\title{
Sous-métis d'Australie. Sort des métis issus d'unions entre Aborigènes et Asiatiques jusque dans les premiers temps de la Fédération
}

Substandard Australian Half-Castes. Fate of Half-Castes of Aboriginal and Asian Descent during the Founding Years of the Commonwealth

\section{Martine Piquet}

\section{OpenEdition}

Journals

Édition électronique

URL : http://journals.openedition.org/rfcb/677

DOI : $10.4000 /$ rfcb. 677

ISSN : 2429-4373

Éditeur

CRECIB - Centre de recherche et d'études en civilisation britannique

Édition imprimée

Date de publication : 15 octobre 2012

Pagination : 103-112

ISBN : 2-911580-37-0

ISSN : 0248-9015

\section{Référence électronique}

Martine Piquet, "Sous-métis d'Australie. Sort des métis issus d'unions entre Aborigènes et Asiatiques jusque dans les premiers temps de la Fédération », Revue Française de Civilisation Britannique [En ligne], XVII-2 | 2012, mis en ligne le 15 mars 2016, consulté le 30 avril 2019. URL : http:// journals.openedition.org/rfcb/677 ; DOI : 10.4000/rfcb.677

\section{Ce document a été généré automatiquement le 30 avril 2019}

\section{c) $(1) \odot$}

Revue française de civilisation britannique est mis à disposition selon les termes de la licence Creative Commons Attribution - Pas d'Utilisation Commerciale - Pas de Modification 4.0 International. 


\title{
Sous-métis d'Australie. Sort des métis issus d'unions entre Aborigènes et Asiatiques jusque dans les premiers temps de la Fédération
}

\author{
Substandard Australian Half-Castes. Fate of Half-Castes of Aboriginal and Asian
} Descent during the Founding Years of the Commonwealth

\section{Martine Piquet}

1 À la proclamation officielle de la Fédération australienne (Commonwealth of Australia) le $1^{\mathrm{er}}$ janvier 1901, à Centennial Park à Sydney, l'un des pères fondateurs et premier Premier ministre fédéral, Edmun Barton, déclarait : « Pour la première fois, nous avons une nation pour un continent et un continent pour une nation ». Dans son esprit, comme dans celui de ses compatriotes, la nouvelle nation-continent devait être une et blanche, un sanctuaire pour la race britannique (a preserve for the British race) dont les colons qui prenaient leur indépendance (en réalité toute relative) représentaient la fine fleur. Tenant l'un des postes les plus avancés de l'Empire, ils se considéraient en effet comme "de meilleurs Britanniques que les Britanniques eux-mêmes» (Better Britons than the Britons themselves) dans leur environnement à leurs yeux plus sain et plus égalitaire que celui d'une Grande-Bretagne victorienne aux classes sociales figées, aux valeurs rigides, aux cités industrielles enveloppées par le smog, où régnait la pauvreté. L'idéal eût été de demeurer entre gens de bonne compagnie. Ainsi, Alfred Deakin, autre père de la Fédération qui allait lui-même devenir Premier ministre quelques années plus tard, affirmait lors de débats parlementaires : «L'unité de la race est une condition absolue à l'unité de l'Australie $»^{1}$. Pour lui, il convenait « [d'interdire] toute immigration étrangère de couleur » et d'organiser "par des moyens justes et raisonnables, le plus rapidement possible, l'expulsion ou la réduction du nombre d'étrangers parmi nous ». Il concluait: «Les deux vont de pair et constituent les éléments indispensables d'une seule et même 
politique - celle qui consiste à garantir la viabilité d'une "Australie blanche" $»^{2}$. Ceci allait être réalisé par la mise en place de tout un appareil législatif, comme on le verra plus loin.

Si les propos de Deakin ne mentionnaient pas les Aborigènes, c'est que leur sort semblait scellé. À l'arrivée des Britanniques à la fin du XVIII e siècle, les populations autochtones étaient peu nombreuses, puisqu'on les estime généralement à environ un demi-million d'individus répartis sur l'ensemble du territoire. Nomades chasseurs-cueilleurs sans notion du travail de la terre tel que le concevaient les Européens, ils n'avaient pas d'organisation sociale identifiable par les nouveaux arrivants, notamment pas de hiérarchie politique ni de structure militaire reconnaissables par eux. Au cours du XIX siècle, succès des théories raciales et darwinisme social aidant, les Aborigènes australiens furent perçus comme une race primitive qui serait incapable de survivre au contact de celle qui, au faite de sa gloire impériale, ne doutait pas d'être la plus évoluée au monde, la britannique. Dès cette époque, entre pitié, parfois sincère, pour des êtres ainsi inexorablement condamnés à disparaître (doomed to die) et malveillance délibérée, plus répandue, envers les occupants de terres où l'on voulait s'installer, et alors que ceux-ci n'avaient pas toujours le bon goût de se laisser faire, les colonies n'avaient pas tardé à concevoir des dispositifs réglementaires et législatifs propres à hâter la réalisation de la prophétie de leur disparition prochaine. On imagina ainsi que les Aborigènes " pur sang " (full blood) devraient être isolés et placés dans des réserves protégées où ils finiraient par s'éteindre naturellement, où, en quelque sorte, on les veillerait (smoothing their dying pillow), alors que les métis «demi-sang ( (half-castes) devraient être acclimatés de force dans la société blanche, quitte à les arracher manu militari à leurs familles et à les placer dans des institutions religieuses ou d'État, voire au sein de familles blanches. En les coupant de tout contact avec leurs communautés d'origine et en ne leur autorisant de mariages qu'avec des blancs ou d'autres métis n'ayant pas un degré d'aboriginalité plus élevé que le leur, on espérait rapidement aboutir à la disparition des Aborigènes par dilution génétique au sein de la race blanche. Contrairement à ce qui était le cas aux États-Unis, on ne craignait pas de retour atavique car le "sang » indigène était considéré comme " primitif » et donc plus « faible » que le sang britannique. Dans les colonies, puis les États et Territoires issus des colonies, des «Protecteurs des Aborigènes» furent chargés de veiller à l'application de la mise sous tutelle des autochtones australiens pour mener à bien cette opération de «blanchiment ».

3 Avec la Fédération, ce qu'on appela, quoique jamais officiellement, "Politique de l'Australie blanche ", allait viser à finir de régler la " question aborigène ", mais surtout celle de l'immigration non européenne. Dès la Fédération proclamée, c'est au nom de cette politique que l'on verrouilla l'accès au pays, que l'on expulsa certains résidents (notamment mélanésiens), que l'on déplaça de force voire que l'on interna certaines catégories de population (dont certains Européens) et, envers les autochtones, que l'on institutionnalisa des pratiques génocidaires, le refus de la citoyenneté et la ségrégation. L'une des toutes premières lois votées par le nouveau Parlement fédéral fut ainsi la Loi de restriction de l'immigration (Immigration Restriction Act) dont le but, comme son nom l'indiquait sans ambiguïté, était de barrer l'entrée du continent aux immigrants jugés «non désirables ». Parallèlement, on mit tout en œuvre pour écarter les non-Européens présents sur le territoire de la vie de la Cité. L'Article 4 de la loi sur le droit de vote fédéral de 1902 (Commonwealth Franchise Act) excluait des listes électorales fédérales, outre les Aborigènes d'Australie, "tout indigène aborigène d'Asie, d'Afrique ou des îles du Pacifique à l'exception de la Nouvelle-Zélande", qui ne jouissait pas du droit de vote dans son État de 
résidence au titre de l'Article 41 de la constitution fédérale. L'année suivante, les mêmes « indigènes aborigènes d'Australie, d'Asie, d'Afrique ou des îles du Pacifique à l'exception de la Nouvelle-Zélande " se voyaient écartés du champ d'application de la loi sur la naturalisation (Naturalization Act 1903). À partir de 1920, l'octroi de la naturalisation releva de «la discrétion absolue » du Gouverneur général, le texte précisant : «il peut, avec ou sans justification explicite, accorder ou retirer le certificat [de naturalisation] en fonction de ce qu'il juge être l'intérêt public, et sa décision est sans appel ». La législation sociale n'était pas davantage applicable aux non-Européens. Ainsi, la Loi sur les pensions d'invalidité et de retraite de 1908 (Invalid and Old Age Pensioners Act) excluait « les Asiatiques " (sauf s'ils étaient nés en Australie) et « les indigènes aborigènes d'Australie, d'Asie, d'Afrique, des îles du Pacifique et de Nouvelle-Zélande ». En 1912, l'État fédéral refusait l'attribution de la prime de maternité aux « Asiatiques ou indigènes natives d'Australie, d'Asie, d'Afrique ou des îles du Pacifique ». De même, dans le domaine de l'emploi, la législation discriminatoire se généralisa entre 1901 et 1920. Dans les secteurs subventionnés par l'État fédéral, comme l'agriculture, l'exploitation minière ou la poste, seul était autorisé l'emploi d'une main-d'œuvre blanche. Au niveau des États, c'est au Queensland et en Australie-Occidentale que la discrimination fut la plus sévère. Au Victoria et en NouvelleGalles du Sud, la législation visait à contrecarrer la «concurrence déloyale » des travailleurs de "couleur", en limitant leurs heures de travail et les soumettant à des contrôles stricts, souvent vexatoires, sur leurs lieux de travail, etc.

On s'aperçoit à la lecture de ces quelques exemples que si les Aborigènes n'étaient pas les seuls à être visés pour des raisons raciales, ils étaient les seuls à figurer dans l'écrasante majorité des législations et réglementations exclusives mises en place tant au niveau des états qu'au niveau fédéral. Être "sujets britanniques » ne leur servait à rien car, selon la définition du "sujet britannique » établie dès le début du XVII ${ }^{e}$ siècle, reprise dans la législation britannique en 1914 puis en Australie dans la loi sur la nationalité de 1920, celui-ci n'était qu'une " personne née dans les possessions du Roi et sous son allégeance " (à l'exception de certaines catégories telles que des enfants de diplomates ou de prisonniers de guerre). Une telle définition couvrait ainsi beaucoup de monde, y compris les Indiens ou les Hongkongais qui étaient exclus de l'accès aux droits de citoyens en Australie. Il n'exista d'ailleurs aucune définition de la "citoyenneté australienne" jusqu'en 1948, et même lorsque le "citoyen australien» devint une entité légale à l'occasion du vote de la Loi sur la nationalité et la citoyenneté (Nationality and Citizenship Act), cela ne changea pas grand chose pour les autochtones. En juin 1949, cinq mois après le vote de la loi, le ministère de la Justice conseilla au ministère de l'Immigration de répondre en ces termes sur le statut des Aborigènes et des « demi-sang 》: "Les indigènes ou les indigènes demi-sang sont [...] des citoyens australiens. Il faut noter cependant que la loi sur la nationalité et la citoyenneté n'a pas pour but en elle-même de modifier les effets de la législation existante sur les droits et devoirs des individus et que la position des indigènes relativement à cette législation n'a pas été modifiée par le seul fait du vote de cette loi ».

$5 \quad$ Les termes des lois d'exclusion votées au début de la Fédération étaient assez larges pour s'appliquer à la plupart des gens de couleur, mais la véritable obsession des Australiens était de fermer leur pays aux « Orientaux ». Une peur irraisonnée du " Péril jaune » leur faisait craindre le déferlement massif de «hordes » en provenance des régions les plus surpeuplées de Chine, alors que la main d'œuvre asiatique déjà sur place était perçue comme déloyalement concurrentielle. Celle-ci était employée dans l'ensemble des colonies sur les grandes exploitations d'élevage (cattle stations), les plantations sucrières, 
dans l'industrie perlière, les transports, les travaux publics ou la domesticité. Dans les années 1850, au moment de la ruée vers l'or, les Chinois avaient afflué sur les champs aurifères, où ils avaient repris avec succès des concessions abandonnées par des Européens moins opiniâtres qu'eux. Plus rarement, ils ouvraient de petits commerces (dont les légendaires blanchisseries) ou s'installaient comme petits exploitants agricoles. Dans le nord, les petits patrons chinois employaient couramment des Aborigènes pour des salaires plus élevés que ceux qu'auraient offerts des Européens. Le plus souvent originaires du sud-est de la Chine, parfois de Singapour, la plupart d'entre eux étaient illettrés, ne parlaient pas l'anglais et ne connaissaient rien de la culture occidentale. C'étaient des travailleurs frugaux, méthodiques, honnêtes et loyaux envers leurs employeurs, mais qui avaient tendance à vivre entre eux, selon leurs propres usages. Leur présence était mal perçue par des colons européens qui nourrissaient des préjugés contre leur prétendue immoralité, notamment parce qu'ils fumaient l'opium. On les accusait des pires crimes dont le rapt d'honnêtes femmes blanches par des réseaux de proxénètes ou encore le vol d'enfants à des fins anthropophagiques. Les dispositions contre les Chinois ne furent pas sans effets puisque leur nombre chuta de 50.000 en 1888 à 32.000 dès 1901 .

6 Pour autant, toutes ces mesures ne résolvaient en rien le problème particulier qui touchait le nord tropical du continent australien, à savoir celui de populations asiaticoaborigènes métissées de longue date. De fait, si l'histoire contemporaine de l'Australie commence avec l'arrivée de la Première Flotte et sa cargaison de bagnards à Sydney Cove en janvier 1788, marquant le début de la colonisation par les Britanniques, les contacts entre autochtones et ultramarins sont bien antérieurs. Il est établi que depuis au moins le $\mathrm{XVII}$ siècle, des commerçants et des pêcheurs macassans venus d'Indonésie visitaient régulièrement la Terre d'Arnhem, au nord du continent, nouant des liens économiques et culturels, apportant avec eux non seulement des marchandises mais aussi de la technologie, des croyances et des cérémonies, s'installant parfois. Il est également très probable que des marins arabes et chinois atteignirent, voire fréquentèrent sporadiquement ces régions bien avant l'arrivée des Européens. Il n'y a pourtant qu'une ou deux décennies que ces éléments sont véritablement pris en compte. Jusque-là, on se focalisait sur les rapports entre Blancs et Aborigènes, et l'on n'accordait qu'un intérêt limité au fait que les autochtones aient pu avoir commerce avec des voisins du nord ou avec des individus non européens arrivés au gré des besoins de la colonisation. Ainsi, un effet d'optique a longtemps fait présenter l'Australie comme un contient isolé du reste du monde depuis les temps les plus reculés, un lieu préservé où flottait encore un parfum de Préhistoire, dont les indigènes, avec leur faciès prognathe, ne pouvaient être que des fossiles vivants, descendants de quelque cousin de Cro-Magnon. En 1982, l'historien Geoffrey Blainey dans son ouvrage intitulé sans la moindre ambiguité The Tyranny of Distance: How Distance Shaped the History of Australia montrait comment le pays avait été façonné par la distance : celle séparant entre elles les régions d'une contrée vaste comme quatorze fois la France, mais celle surtout la séparant de la Grande-Bretagne, voire plus largement du monde occidental: Londres est à $17.000 \mathrm{~km}$ de Sydney. Or il suffit de regarder une carte régionale pour s'apercevoir que le Cap York, au nord du continent australien n'est séparé de la Papouasie-Nouvelle Guinée que par les quelque 150 kilomètres du Détroit de Torres, et que la capitale du Territoire-du-Nord, Darwin, est moins éloignée de Jakarta (un peu plus de $2700 \mathrm{~km}$ ) que de la capitale fédérale, Canberra, ou des deux grandes métropoles du sud, Sydney et Melbourne (un peu moins de $3150 \mathrm{~km}$ ). La capitale d'État la plus proche, Brisbane, est à un peu plus de $1750 \mathrm{~km}$ quand Dili, la ville indonésienne d'importance la plus proche, est distante d'à peine $720 \mathrm{~km}$. 
Pourtant, cette proximité d'avec l'Asie du sud-est a longtemps été délibérément ignorée au profit d'un tropisme maladif vers l'ancienne mère-patrie. Il est vrai que la colonisation britannique s'est effectuée à partir du sud-est vers le nord et l'ouest plutôt qu'à partir du nord, ceci expliquant plus que probablement cela.

Pour ces raisons de proximité géographique avec l'Asie et, inversement, d'éloignement des grands centres de colonisation britannique plutôt situés dans les régions côtières du sud-est, les rencontres, échanges et mariages entre Asiatiques et Aborigènes furent surtout fréquents dans le nord du continent, plus particulièrement le Territoire-du-Nord et l'Australie-Occidentale, mais aussi le Queensland. Dans ces régions septentrionales éloignées des régions plus peuplées du sud et de l'est, les Européens restèrent minoritaires jusqu'à la seconde guerre mondiale. Malgré le sentiment anti-asiatique très largement partagé décrit plus haut, la rareté de la main d'œuvre fit que l'on se vit contraint de continuer à recruter Chinois, Japonais, Philippins, Malais, Afghans... dans les secteurs essentiels au développement de la colonisation du nord du continent australien : l'industrie pastorale (surtout au Queensland), l'industrie perlière (principalement l'Australie-Occidentale) et le transport (chemins de fer dans le Territoire-du-Nord, transport de marchandises à dos de chameau à travers les régions désertiques, etc.). L'infériorité numérique européenne ne manquait pas de frapper les visiteurs qui ne pouvaient que constater la vigueur de la concurrence asiatique au niveau des entreprises locales mais, bien pire, celle d'un développement démographique métissé, qui contredisait les oracles évolutionnistes et montrait les limites de la politique de l'Australie blanche. On avait là le pire mélange imaginable, celui précisément des groupes que l'on voulait voir disparaître du pays. Ni la législation ciblant les immigrants asiatiques ni celle relative à l'administration des populations autochtones ne faisaient sens pour cette population largement métissée. On tâcha donc de limiter les rapprochements entre Asiatiques et Aborigènes, souvent sous des prétextes prophylactiques où le moral le disputait au médical. La «malpropreté », la « lascivité » et la «paresse" qu'on prêtait aux Asiatiques étaient désignées responsables de la propagation de maladies telles que la variole ou la lèpre, ou encore des problèmes d'addiction à l'opium. En 1901, au Queensland, par exemple, la Loi de protection des Aborigènes de 1897 qui prévoyait déjà l'interdiction de vente d'opium aux autochtones fut renforcée par un amendement interdisant aux Chinois d'employer des indigènes. En 1905, en Australie-Occidentale, la Loi de Protection locale interdit tout contact entre femmes aborigènes et pêcheurs de perles japonais. Les Protecteurs des Aborigènes, dont cela entrait dans les attributions, opposaient quasi systématiquement leur veto aux demandes de mariages mixtes entre Aborigènes et Asiatiques (et non-Aborigènes plus généralement, Mélanésiens en particulier, par exemple au Queensland dès 1890). Au sein de ces populations métissées, l'une des difficultés auxquelles ils étaient confrontés était la difficulté à distinguer les "Aborigènes » des "non-Aborigènes ", les " Asiatiques " des «non-Asiatiques ». On était par ailleurs fort embarrassé par l'appellation " demi-sang " ( half-castes) que l'on avait plutôt l'habitude d'employer pour les enfants issus d'une mère aborigène et d'un père blanc, d'où l'apparition d'une catégorisation "ni Aborigène ni demi-sang " qui reléguait les intéressés au rang de parias. Pour autant, la confusion et l'arbitraire régnaient. La catégorisation variait selon l'endroit où l'on était né, la date à laquelle on avait vu le jour et la discrétion des autorités. Les membres d'une même fratrie, issus des mêmes parents, pouvaient se trouver placés dans des catégories différentes et une même personne pouvait voir sa catégorisation modifiée plusieurs fois. 
8 La définition de l'aboriginalité elle-même ne fut jamais claire. On a vu dans les premières lois votées au moment de la Fédération des colonies australiennes l'importance accordée à la notion "d'indigène aborigène " (aboriginal native). Sous ses dehors redondants, cette expression était spécifiquement australienne au sein de l'Empire. En effet, les Britanniques n'utilisaient pas les mots «aborigine» et «aboriginal » pour désigner les populations indigènes de leurs colonies, préférant le terme de "native ». Dans l'usage australien les mots « aboriginal » et «native» s'appliquaient à deux types bien distincts de population, comme l'avait d'ailleurs noté Trollope en son temps ${ }^{4}$, le premier désignant les autochtones, avec le sens d'habitant ou descendant d'habitant originel d'une contrée, tandis que le second désignait les colons blancs nés en Australie, ce qu'on aurait appelé un «créole » en Louisiane ou dans la Caraïbe française. Le mot «native» en Australie renvoyait donc au lieu de naissance tout autant qu'à l'origine ethnique proprement dite. Ainsi, malgré son apparence pléonastique, le terme "aboriginal native » était à lire en fonction de l'étymologie de ses composantes et désignait le descendant du peuple originel d'une région (aboriginal) né dans cette région (native). Or si la notion "d'indigénéité " ainsi définie ne posa jamais problème, il en alla tout autrement de la définition de «l'aboriginalité » en raison des enjeux qui y étaient liés : la ligne de démarcation entre qui était «aborigène » et qui ne l'était pas conditionnait en effet l'exclusion ou non des droits civiques et des prestations sociales. La question était cruciale pour les « demisang ", qui se trouvaient ballottés d'un côté à l'autre de la frontière pour eux très fluctuante de l'accession à la citoyenneté, mais elle concernait aussi les immigrés de couleur requérant la naturalisation, l'inscription sur les listes électorales ou l'accès aux prestations sociales.

C'est d'ailleurs à propos de ces derniers que se posa rapidement la question, quand il fallut déterminer le statut des «indigènes aborigènes d'Asie, d'Afrique et du Pacifique ». Un avis donné en 1904 au ministère de l'Intérieur par le Conseil juridique de la Couronne sur la nationalité d'enfants nés en Australie de parents asiatiques afin de savoir s'il y avait lieu ou non de les inscrire sur les listes électorales arguait que «les personnes nées dans l'Empire britannique sont des sujets britannique - quelle que soit la nationalité de leurs parents". On appliquait ainsi strictement la loi du sol puisque le terme native se rapportait au lieu de naissance, quels que fussent la nationalité et le lieu de naissance des parents. Pourtant, la même année, le Conseil donna un avis exactement contraire dans une affaire de naturalisation. Il s'agissait du cas d'un certain R.A. Salleeby, d'origine syrienne et né à New York. Sa demande de naturalisation avait été rejetée au motif que l'intention du parlement avait été de "refuser le privilège de la naturalisation aux personnes d'ascendance asiatique ». L'intéressé interjeta appel, alléguant (1) que les Syriens modernes n'étaient plus les Syriens des origines, (2) que ses ancêtres descendaient des Croisés et (3) qu'il était par ailleurs admis que les Syriens modernes étaient de race caucasienne. Cette argumentation plongea les autorités dans le plus grand embarras, puisque le requérant tentait de faire valoir une interprétation littérale et étroite du terme aboriginal. Lui donner raison aurait rendu les dispositions de la loi inapplicables. Pour éviter cet écueil, l'avis fut donné que, dans le cadre de la loi sur la naturalisation aboriginal native désignerait " tout indigène appartenant à une "race indigène " d'Asie, d'Afrique, etc. » Dans cette acception large, aboriginal native en venait à désigner tout non-Européen.

Cet avis fut confirmé en 1923 dans un cas similaire, sur lequel eut à se prononcer la Haute Cour fédérale ${ }^{5}$, où un Japonais, Jiro Murramats, né au Japon de parents japonais, naturalisé au Victoria en 1899 et installé en Australie-Occidentale depuis 1900, n'obtenait 
pas son inscription sur les listes électorales fédérales, alors qu'il avait été inscrit sans difficulté sur les listes électorales de cet État qui pourtant ne reconnaissait pas le droit de vote aux « indigènes aborigènes d'Australie, d'Asie, d'Afrique ou des îles du Pacifique, ni au personnes de demi-sang ». Comme le Syrien R.A. Salleeby avant lui, Jiro Murramats avait tenté de faire valoir qu'il ne descendait pas des premiers habitants du Japon, les Ainus, mais de colons plus tardifs. Dans les attendus de son jugement, la Haute Cour indiqua "qu'il ne suffisait pas [au plaignant] de montrer que sa race n'était pas une race "aborigène" du Japon, il fallait qu'il montre qu'il n'appartenait pas à une race aborigène d'Asie ou des Îles du Pacifique ». Pour la Haute Cour, « aborigène » signifiait :

[...] aborigène dans le sens commun du terme : ceux qui appartiennent à la race qui habitait un pays au moment où les Européens y sont arrivés. Il se peut, comme certains le prétendent, qu'il ait existé une race peuplant l'Australie avant ceux que nous appelons «les aborigènes australiens » (...); il se peut qu'avant que les Japonais arrivent au Japon il y ait eu une race appelée « les Ainus », et même avant eux des hommes des cavernes. Mais cela n'empêcherait pas les Noirs australiens d'êtres les aborigènes d'Australie du point de vue des colons blancs, de la loi australienne ni les Japonais actuels d'être les aborigènes du Japon.

11 Ces interprétations ne furent pas sans affecter les métis d'ascendance aborigène et non européenne pour leur accession à la citoyenneté. Comme pour l'appréciation de l'opportunité d'accorder l'autorisation des mariages mixtes, une situation similaire pouvait être jugée complètement différemment. Ainsi un insulaire de l'île Thursday, de père originaire des îles Loyauté naturalisé australien et de mère insulaire " pur sang » du Détroit de Torres, obtint sans difficulté son inscription sur les listes électorales, puisqu'on considéra que la naturalisation de son père suffisait à faire de lui un «demi-sang ». Au contraire, à Broome en Australie-Occidentale, une femme de mère aborigène "pur sang " et de père " présumé » originaire des Philippines mais non naturalisé, se vit refuser son inscription sur les listes électorales au motif qu'étant «moitié philippine» et «moitié aborigène ", donc n'ayant pas de sang européen, elle ne pouvait être considérée comme «demi-sang ». Cette aboriginalité à géométrie variable pouvait s'appliquer à un seul et même individu En Australie-Méridionale, par exemple, les « demi-sang » étaient assujettis à la conscription militaire mais pas les "pur-sang ». Pourtant les « demi-sang » étaient souvent classés avec les "pur-sang» dans d'autres cas. Au niveau fédéral, un "demisang » incapable de subvenir à ses besoins pouvait être rétrogradé au rang de "pur-sang pour être placé sous tutelle mais, en tant que «demi-sang", il conservait son droit de vote.

12 Malgré les efforts pour tenter de fixer la définition de «l'aboriginalité », celle-ci resta largement à la discrétion des autorités locales jusque dans les années 1950. La seule certitude était qu'elle se déclinait alors en termes de "sang" plutôt que d'identité culturelle. Pourtant, contrairement aux Américains, Canadiens ou Sud-Africains, les autorités australiennes refusèrent d'adopter un système complexe de classification sanguine distinguant "mulâtre» (mulatto), "quarteron" (quadroon), "octavon » (octavoon), etc. On préféra se cantonner au principe plus simple, implicite depuis 1901, que toute personne ayant moins de $50 \%$ de «sang blanc» serait considérée comme « indigène ». Ainsi, les « demi-sang » devenaient des «Européens » dans la plupart des cas, sauf lorsqu'il arrangeait les autorités qu'il en fût autrement. Les métis «ni Aborigènes ni demi-sang ", pour leur part, devaient rester pour longtemps aux marges de la société australienne, des "étrangers de l'intérieur » pour reprendre l'expression de Peta Stephenson' ${ }^{6}$. La définition de l'aboriginalité demeure sujet d'âpres débats, mais on 
considère aujourd'hui qu'est Aborigène "toute personne d'origine aborigène s'identifiant comme telle et reconnue par la communauté aborigène au sein de laquelle elle vit ${ }^{7}$ ", ce qui laisse enfin toute leur place aux métis de toutes ascendances.

\section{BIBLIOGRAPHIE}

CHESTERMAN John et GALLIGAN Brian, Citizens without Rights : Aborigines and Australian Citiznship, Cambridge University Press, Cambridge, 1997.

EDWARDS Penny \& YUANFANG Shen (dir.), Lost in the Whitewash. Aboriginal-Asian Encounters in Australia, 1901-2001, ANU, 2003.

GANTER, Regina, Mixed Relations. Asian-Aboriginal Contact in North Australia, University of Western Australia Press, 2006.

HAVEMANN Paul (dir.), Indigenous Peoples' Rights in Australia, Canada \& New Zealand, Oxford UniversityPress New Zealand 1999.

POVINELLI Elizabeth A., The Cunning of Recognition. Indigenous Alterities and the Making of Australian Multiculturalism, Duke University Press, Durham \& London, 2002.

STEPHENSON, Peta, The Outsiders Within. Telling Australia's Indigenous-Asian History, University of Ne South Wales Press, Sydney, 2007.

\section{NOTES}

1. Commonwealth Parliamentary Debates, 12 septembre 1901, vol. iv, pp. 4.804-07.

2. Commonwealth Parliamentary Debates, 12 septembre 1901, ibid.

3. "Aborigines or half caste aborigine are [...] Australian citizens. It is pointed out, however, that the Nationality and Citizenship Act does not itself purport to alter the effect of existing legislation upon the rights and duties of individuals, and the position of aborigines in relation to such legislation has not been altered solely by reason of the provision of that Act."

4. "It will be as well to call the race by the name officially given to it. The government styles them "aboriginals" ... the word native is almost universally applied to white colonists born in Australia.", Anthony TROLLOPE, Australia and New Zealand, Melbourne, Authorized Australian Edition, 1873, pp. 504.

5. Haute Cour : rôle équivalent à celui de la Cour suprême américaine.

6. Titre de son ouvrage The Outsiders Within, 2007.

7. Report on a review of the administration of the working definition of Aboriginal and Torres Strait Islanders (Canberra, 1981) : "An Aboriginal or Torres Strait Islander is a person of Aboriginal or Torres Strait Islander descent who identifies as an Aboriginal or Torres Strait Islander and is accepted as such by the community in which he (she) lives". 


\section{RÉSUMÉS}

Pendant une bonne partie de sa courte histoire et particulièrement aux cours des années fondatrices du Commonwealth, l'Australie se projetait comme une nation «britannique » des Antipodes. L'idéal de la "politique de l'Australie Blanche» eût été de limiter l'accès de son territoire aux seuls immigrants originaires des îles britanniques, de diluer génétiquement comme culturellement la minorité aborigène. Pourtant, le monde changeant, l'Australie finit, bien à contrecœur, par se résoudre à accepter l'arrivée croissante d'Européens de l'Est et du Sud, puis d'Asiatiques. Le rapport Bringing Them Home de 1997 sur les « Générations volées » d'enfants arrachés à leurs foyers pour être « civilisés » dans des institutions religieuses ou d'État a dénoncé l'un des aspects peu glorieux du traitement réservé aux métis. Il était là question surtout de métis issus d'unions entre blancs et Aborigènes. Or quoi qu'eussent voulu oublier et/ou faire oublier les Australiens, il existait avant même l'installation des premiers colons européens des relations entre les Aborigènes et leurs voisins du nord, et il y eut une immigration asiatique non négligeable dès le XIX ${ }^{\mathrm{e}}$ siècle. En raison de leur double ascendance, les métis issus d'unions entre Asiatiques et Aborigènes se trouvèrent doublement stigmatisés par l'addition des préjugés raciaux. Leurs difficultés spécifiques à accéder à la citoyenneté jusqu'au $\mathrm{XX}^{\mathrm{e}}$ siècle sont particulièrement révélatrices $\mathrm{du}$ profond rejet de l'altérité qui a caractérisé la société australienne jusqu'à l'adoption officielle du multiculturalisme dans les années 1970.

For a long period of its short history, most particularly during the founding years of the Commonwealth, Australia thought of itself as a "British" nation of the Antipodes. Ideally, the socalled "White Australia Policy" was meant to bar the access of its territory to immigrants other than from the British Isles, and to gradually dilute the Aboriginal minority, both genetically and culturally into white society. This, however, eventually proved impossible to achieve as times were changing and Australia increasingly found it had no choice but to let come the immigrants it needed from other parts of the world (Eastern and Southern Europe, then Asia). The 1997 Bringing Them Home Report on the "Stolen Generations" of children forcibly removed from their families to be "civilised" by religious or state institutions illustrated one of the most scandalous aspects of the way in which half-caste children were treated. Most of those were children with a white parentage. However, and although those facts were often more or less deliberately ignored until recently, Aboriginal people had entertained contacts with their northern neighbours before the arrival of the first white settlers, and there had been a substantial Asian immigration since the $\mathrm{XIX}^{\text {th }}$ century. Half-castes of Aboriginal and Asian descent were doubly stigmatised by the prejudices against both races. The specific difficulties they met before they could be regarded as citizens are very typical of the deep reject of otherness among Australian society, until the official adoption of multicultural policies in the 1970 s.

\section{AUTEUR}

\section{MARTINE PIQUET}

Martine Piquet est Professeur à l'Université Paris-Dauphine où elle dirige le CICLaS EA 4405. Elle est spécialisée dans l'étude de la civilisation des Pays du Commonwealth et plus particulièrement 
celle d'Australie à laquelle elle a consacré de nombreux articles et l'ouvrage Australie Plurielle, l'Harmattan, 2004. Elle co-dirige les revues Cultures of the Commnwealth (ISSN 1245-2971) et Les Cahiers du CICLaS (ISSN 1637-7060). 